\title{
INTRODUCTION TO THE SPECIAL ISSUE ON PRINCIPLES AND PRACTICES IN MULTI-AGENT SYSTEMS
}

Agent-based Computing addresses the challenges in managing distributed computing systems and networks through monitoring, communication, consensus-based decision-making and coordinated actuation. As a result, intelligent agents and multi-agent systems have demonstrated the capability to use intelligence, knowledge representation and reasoning, and other social metaphors like 'trust', 'game' and 'institution', to not only address real-world problems in a human-like way but to transcend human performance. This has had a transformative impact on many application domains, particularly e-commerce, but also on planning, logistics, manufacturing, robotics, decision support, transportation, entertainment, emergency relief \& disaster management, and data mining \& analytics. As one of the largest and still growing research fields of Computer Science, agent-based computing today remains a unique enabler of inter-, multi- and trans-disciplinary research.

This special issue provides a selection of papers concerning the state-of-the-art research in multi-agent systems. Although the idea was initiated at 17th International Conference on Principles and Practice of MultiAgent Systems, an open call allowed any researcher working on related topics to submit a paper for review.

This special issue features four articles:

- The article entitled "Combining PosoMAS Method Content with Scrum: Agile Software Engineering for Open Self-Organising Systems" by Jan-Philipp Steghöfer, Hella Seebach, Benedikt Eberhardinger, Michael Hübschmann, Wolfgang Reif proposes PosoMAS-Scrum, an agile agent-oriented software engineering process for developing large-scale open self-organising systems. The authors also demonstrate how this method can be applied in a development effort and compare it with existing approaches.

- The article entitled "Modelling Dynamic Normative Understanding in Agent Societies" by Christopher Konstantin Frantz, Martin K. Purvis, Bastin Tony Roy Savarimuthu, Mariusz Nowostawski proposes an approach to build up normative understanding from the bottom up without using any prior knowledge. Their approach combines both existing institution representations (the structure) with a norm identification process

- The article entitled "A Multi-Agent Approach for Trust-based Service Discovery and Selection in Social Networks" by Amine Louati, Joyce El Haddad, Suzanne Pinson proposes an approach to use multi-agent systems to model the service discovery and selection process. The authors present a trust model for social networks which supports agents in determining trustworthy service providers and allows agents to reason about trust in their distributed decision-making process.

- The article entitled "Multi-Objective Distributed Constraint Optimization using Semi-Rings" by Graham Billiau, Chee Fon Chang and Aditya Ghose proposes an extended Support Based Distributed Optimization algorithm to support Multi-Objective Distributed Constraint Optimization problem. The authors also demonstrate that by building a new DCOP definition using an idempotent semiring to measure the cost/utility of a solution, this approach is able to solve multiple objectives without a total pre-order over the set of solutions.

We would like to thank the editorial board of SCPE for the chance of arranging this special issue, and all the reviewers for their hard work.

Hoa Khanh Dam, Jeremy Pitt, Guido Governatori, Takayuki Ito, Yang Xu. 
